\title{
Regression Analysis for Soft Rock Tunnel Monitoring Data Based on Data-Mining by SPSS
}

\author{
TANG Jiejun \\ Hunan Communication Polytechnic College, Changsha 410132 P.R.China \\ tjjhpq@yahoo.com.cn
}

Keywords: SPSS, vault sink, surrounding convergence, regression analysis, soft rock tunnel

\begin{abstract}
Using SPSS software for pretreated soft rock tunnel, a regression analysis of the monitoring data reflected the internal relationship and change rule between tunnel vault sink and the surrounding convergence and distance from tunnel face to monitoring section and measuring time. The results show that: the regression model of soft rock tunnel fitted well to monitoring data, under different adjacent rock condition the corresponding tunnel deformation model has big difference, according to the fitting regression equation of tunnel monitoring data, we can preliminary calculate the limit value of tunnel excavation after excavating and the time needed until stable state to provide strong basis for adjusting performed deformation and later arrangement.
\end{abstract}

\section{Introduction}

In New Orleans method of tunnel construction, the sink of tunnel vault and surrounding convergence must be measured in tunnel monitoring project, which reflect the changing of the surrounding rock stress state[1]. Because field measurement of the original data collected has certain discrete and contingency, it is difficult to obtain effective information before mathematical method analysis[2]. Therefore, it is very important to do statistical analysis of the tunnel monitoring data and find out the inner relationship between the data and the change rule. The regression analysis in monitoring data analysis is used most widely[3].

SPSS(Statistical Package for the Social Science) software is used widely in communication, medical care, bank, market research, scientific research education, and other fields and industry because of it is clear, direct, easy to use, powerful graphics function characteristic and covers a wide range of contents[4].In this paper, multiple regression analysis by SPSS software is done to simulate mathematically the tunnel monitoring data, the distance of the tunnel face to monitoring section, and find out some rules and change, so as to guide engineering construction.

\section{Multiple regression analysis theory}

Multiple regression analysis is the study theory and method of the relationship between a dependent variable and several independent variables. Taking $p$ independent variables into consider, the multiple regression equation can be expressed as[2]:

$$
y_{i}=a+\sum_{j=1}^{p} b_{j} x_{j i}+\varepsilon_{i}
$$

where: $\varepsilon_{i} \sim N\left(0, \sigma^{2}\right)$ is independent identically distributed normal distribution random variables, $a, b_{1}, \ldots, b_{p}$ is regression coefficient.

Regression coefficient $a$ and $b_{j}(j=1,2, \ldots, p)$ is obtained by method of least squares, that is by selecting $a$ and $b_{j}$ we get the least error sum of squares:

$$
S S E=\sum_{i=1}^{n}\left(y_{p}-\hat{y}_{p}\right)^{2}
$$

where: $\hat{y}_{p}=a+\sum_{j=1}^{p} b_{j} x_{j i}, i=1,2 \cdots \cdots \cdot n$.

According to the maximum principle mathematical analysis, to make the SSE minimum, only to 
respectively complete partial derivative to $a$ and $b_{j}$ by equation (2), that is $a$ and $b_{j}$ should meet the following equation:

$$
\left\{\begin{array}{l}
\frac{\partial S S E}{\partial a}=0 \\
\frac{\partial S S E}{\partial b_{j}}=0
\end{array} \quad j=1,2, \ldots, p\right.
$$

Normal equations are got:

$$
\left\{\begin{array}{c}
n a+b_{1} \sum_{i=1}^{n} x_{1 i}+b_{2} \sum_{i=1}^{n} x_{2 i}+\cdots \cdots b_{p} \sum_{i=1}^{n} x_{p i}=\sum_{i=1}^{n} y_{i} \\
a \sum_{i=1}^{n} x_{1 i}+b_{1} \sum_{i=1}^{n} x_{1 i}{ }^{2}+b_{2} \sum_{i=1}^{n} x_{1 i} \cdot x_{2 i}+\cdots \cdots b_{p} \sum_{i=1}^{n} x_{1 i} \cdot x_{p i}=\sum_{i=1}^{n} x_{1 i} y_{i} \\
a \sum_{i=1}^{n} x_{2 i}+b_{1} \sum_{i=1}^{n} x_{1 i} x_{2 p}+b_{2} \sum_{i=1}^{n} x_{2 i}{ }^{2}+\cdots \cdots b_{p} \sum_{i=1}^{n} x_{2 i} \cdot x_{p i}=\sum_{i=1}^{n} x_{2 i} y_{i} \\
\cdots \cdots \cdots \cdots \cdots \cdots \cdots \\
a \sum_{i=1}^{n} x_{p i}+b_{1} \sum_{i=1}^{n} x_{p i} x_{1 i}+b_{2} \sum_{i=1}^{n} x_{p i} \cdot x_{2 i}+\cdots \cdots b_{p} \sum_{i=1}^{n} x_{p i}^{2}=\sum_{i=1}^{n} x_{p i} y_{i}
\end{array}\right.
$$

However the above formula is too multifarious, and the application and derivation is not easy. In order to simplify the expression, matrix representation can be introduced. Here:

$$
\begin{array}{rlrl}
Y & =\left[\begin{array}{l}
y_{1} \\
y_{2} \\
\vdots \\
y_{n}
\end{array}\right] \quad X=\left[\begin{array}{ccccc}
1 & x_{11} & x_{21} & \cdots & x_{p 1} \\
1 & x_{12} & x_{22} & \cdots & x_{p 2} \\
& & \cdots \cdots & & \\
1 & & x_{2 n} & \cdots & x_{p n}
\end{array}\right] \\
B & =\left[\begin{array}{c}
b_{0} \\
b_{1} \\
\vdots \\
b_{p}
\end{array}\right] & \varepsilon=\left[\begin{array}{c}
\varepsilon_{1} \\
\varepsilon_{2} \\
\vdots \\
\varepsilon_{n}
\end{array}\right]
\end{array}
$$

Through a series of operations, finally the regression model can be expressed as:

$$
B=\left(X^{\prime} X\right)^{-1} X^{\prime} Y
$$

\section{Tunnel monitoring data regression model}

In 1979, Panet and Guadin use convergence limit method to analyze the relationship between tunnel vault sink (peripheral convergence) and tunnel face promote distance, and reached a tunnel deformation calculation empirical formula, it is as:

$$
\delta(x)=\delta_{\infty x}\left\{1-[X /(x+X)]^{2}\right\}
$$

Where: $\delta(x)$ is tunnel deformation that is the boundary value of tunnel vault sink or peripheral convergence, $\delta_{\infty x}$ is the boundary value of tunnel vault sink or peripheral convergence due to impelling of tunnel face, $\mathrm{X}$ is the influence distance of tunnel face which is related to the plastic radius of surrounding rock $R_{0}$ and can be calculated by:

$$
X=0.84 R_{0}
$$

To soft rock tunnel, the monitoring section close enough to tunnel face, vault sink (peripheral convergence) value is the co-action of tunnel face promoting distance and rock flow effect. When tunnel face push forward to far enough away from monitoring section, project support effect vanished, the surrounding rock flow effect will become the main influencing factors of tunnel convergence. Based on this, in 1987 Sulem according to the research results of Panet proposed deformation theory of tunnel excavation time-space effect that is soft rock tunnel deformation convergence value is made up of the space effect of tunnel face promoting distance and time effect of surrounding rock [5], its expression is for:

$$
\delta(x, t)=\delta_{\propto x}\left[1-(X /(x+X))^{2}\right]\left\{1+m\left[1-(T /(t+T))^{n}\right]\right\}
$$


Where: $\mathrm{X}$ is the influence distance of tunnel face, $\mathrm{T}$ is the flow characteristic parameters of surrounding rock, $\delta_{\infty x}$ is the instantaneous convergence limit produced by impelling of tunnel face not considering the flow characteristic, $m$ and $n$ is constant.

\section{Example and results analysis}

A tunnel with single hole is taking for example. The starting mileage pile number is ZK2112+285, the ground elevation is $161.18 \mathrm{~m}$, and design elevation is $157.27 \mathrm{~m}$, the bottom depth is 3.91m. The ending pile number is ZK2113+615,the ground elevation is $199.1 \mathrm{~m}$, and design elevation is $192.65 \mathrm{~m}$, the bottom depth is $6.45 \mathrm{~m}$, the total length is $1330 \mathrm{~m}$. Tunnel monitoring section ZK2113+515 is full of strong decency limestone, dolomitic limestone, carst and developed crack, where the surface is of strong corrosion phenomena, without the ability to rock steady, easy to take disintegration, deformation and argillization in water. The arch department and the lateral wall is easy to collapse. The surrounding rock level is level IV.

On the primary tunnel support three vault sink measuring points, three surrounding convergence lines are arranged, as shown in figure 1.

Section ZK2113+515 is selected to be a basic sample of multiple regression analysis, the vault sink $(\mathrm{G})$ as the dependent variable, the distance of tunnel face from monitoring cross-section (L) and measuring time ( $\mathrm{T}$ ) as the independent variable. Because of surrounding rock tunnel monitoring section belongs to soft rock, the soft rock tunnel vault sink regression model is built(verified and modified through following computing). Input the regression equation in the Model Expression, the editor process of soft rock tunnel after vault sink regression model is shown in figure 2.

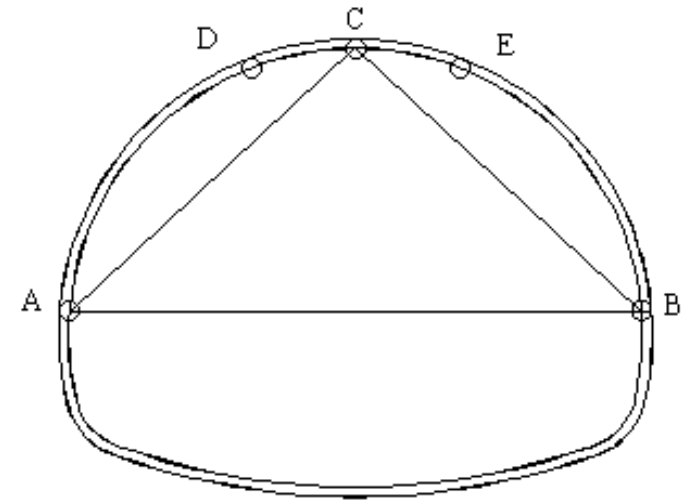

Fig. 1 The arrangement of tunnel deformation measuring points

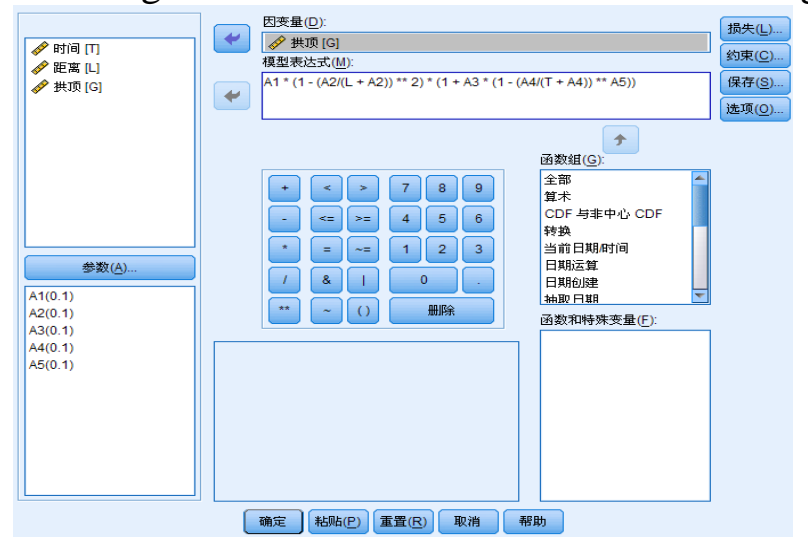

Fig. 2 The vault sink regression model deposed by Model Expression

After deposed by Model Expression, corresponding parameters should be set. In this example initial parameter is as: $\mathrm{A} 1=0.1, \mathrm{~A} 2=0.1, \mathrm{~A} 3=0.1, \mathrm{~A} 4=0.1, \mathrm{~A} 5=0.1$.

Due to the large amount of data, only 17 times iterative calculation results is selected and shown as table 1.

Table 1. Iterative calculation results of soft rock tunnel vault sink

\begin{tabular}{|l|c|c|}
\hline Iteration & Residual & parameter \\
\hline
\end{tabular}




\begin{tabular}{|c|c|c|c|c|c|c|}
\hline number & $\begin{array}{c}\text { sum of } \\
\text { squares }\end{array}$ & A1 & A2 & A3 & A4 & A5 \\
\hline 0.3 & 33554.091 & 0.100 & 0.100 & 0.100 & 0.100 & 0.100 \\
\hline 1.2 & 28658.433 & -2.344 & 0.100 & -0.003 & 0.113 & 0.024 \\
\hline 2.1 & 26875.659 & -3.777 & 0.100 & 0.557 & 0.114 & -0.036 \\
\hline 3.3 & 1478.914 & -9.711 & 0.100 & 2.882 & 0.144 & 0.212 \\
\hline 4.2 & 902.606 & -10.062 & 2.709 & 3.129 & 0.142 & 0.234 \\
\hline 5.1 & 619.43 & -10.475 & 6.035 & 3.431 & 0.243 & 0.255 \\
\hline 6.1 & 596.231 & -10.500 & 5.378 & 3.474 & 0.326 & 0.253 \\
\hline 7.1 & 226.139 & -10.244 & 7.439 & 3.339 & 0.266 & 0.241 \\
\hline 8.1 & 123.071 & -10.286 & 9.726 & 3.393 & 0.268 & 0.244 \\
\hline 9.1 & 64.178 & -10.377 & 12.365 & 3.482 & 0.282 & 0.249 \\
\hline 10.1 & 51.027 & -10.438 & 13.925 & 3.54 & 0.297 & 0.252 \\
\hline 11.1 & 49.160 & -10.468 & 14.637 & 3.568 & 0.308 & 0.253 \\
\hline 12.1 & 49.022 & -10.477 & 14.791 & 3.576 & 0.313 & 0.253 \\
\hline 13.1 & 48.945 & -10.485 & 14.822 & 3.582 & 0.319 & 0.253 \\
\hline 14.1 & 48.651 & -10.517 & 14.785 & 3.605 & 0.348 & 0.252 \\
\hline 15.1 & 47.895 & -10.600 & 14.449 & 3.664 & 0.428 & 0.249 \\
\hline 16.1 & 46.805 & -10.767 & 13.516 & 3.781 & 0.598 & 0.241 \\
\hline
\end{tabular}

Bootstrap method is used to randomly calculate parameters estimation value, standard deviation and 95\% confidence interval of the soft rock tunnel vault sink after 50 times iterative calculations, results are shown in table 2 . The estimate of the parameters were $\mathrm{A} 1=9.201, \mathrm{~A} 2=6.608, \mathrm{~A} 3=$ 6.320, A4 $=2.994$, A5 $=0.213$. Finally soft rock tunnel vault sink regression model can be expressed as

$$
G=(-9.201) \cdot\left[1-\left(\frac{6.608}{L-6.608}\right)^{2}\right] \cdot\left[1+6.320 \cdot\left(1-\frac{2.994}{T+2.994}\right)^{0.213}\right]
$$

where: $G$ is the soft rock tunnel vault sink value, $L$ is the distance from tunnel face to survey section, and $T$ is monitoring time.

Table 2 Calculate parameters estimation value and confidence interval of regression model

\begin{tabular}{|c|c|c|c|c|}
\hline \multirow{2}{*}{ parameter } & \multirow{2}{*}{ Estimate value } & \multirow{2}{*}{$\begin{array}{l}\text { Standard } \\
\text { deviation }\end{array}$} & \multicolumn{2}{|c|}{ 95\% Confidence interval } \\
\cline { 4 - 5 } & & 11.090 & -31.849 & 13.447 \\
\hline A1 & -9.201 & 6.887 & -7.457 & 20.673 \\
\hline A2 & 6.608 & 13.068 & -20.368 & 33.008 \\
\hline A3 & 6.320 & 8.911 & -15.205 & 21.194 \\
\hline A4 & 2.994 & 0.670 & -1.156 & 1.582 \\
\hline A5 & 0.213 & Uper bound & Upper bound \\
\hline
\end{tabular}

After regression model parameters are calculated the parameters are still needed to be evaluated to determine the model relevance and fitting degree. Regression model parameters variance analysis and decision coefficient calculation results are shown as table 3. It can be seen from table 3 decision coefficient is as high as 0.986 , which shows well fitting effect of regression model. Further it verified that using the soft rock tunnel deformation regression model to fitting vault sink monitoring data is reasonable.

Table 3 The parameter variance and confidence interval of regression model

\begin{tabular}{|c|c|c|c|}
\hline Data & Sum of squares & Degree & Sample mean-square \\
\hline Regression & 33301.089 & 5 & 6660.218 \\
\hline Residual err & 35.552 & 30 & 1.185 \\
\hline Total error without amendment & 33336.641 & 35 & \\
\hline Total error with amendment & 2465.867 & 34 & \\
\hline
\end{tabular}

Similarly, we can get soft rock tunnel surrounding convergence regression model as: 


$$
S=(-3.253)\left[1-\left(\frac{8.948}{L-8.948}\right)^{2}\right] \cdot\left[1+8.704 \cdot\left(1-\frac{4.313}{T+4.313}\right)^{0.398}\right]
$$

where: $S$ is the convergence value of soft rock tunnel surroundings, $L$ is the distance from tunnel face to survey section, and $T$ is monitoring time.

\section{Conclusions}

Under different adjacent rock condition the corresponding tunnel deformation model has big difference. So it is important to select proper regression model of the monitoring data for regression analysis. This provides a new solution for future monitoring data regression analysis. According to the regression results of different adjacent rock monitoring data, we can preliminary calculate the limit value of tunnel excavation after excavating and the time needed until stable state, to provide strong basis for adjusting performed deformation and the later arrangement, which can be applied in similar tunnel construction.

\section{References}

[1] Li Xiaohong. New Austrian Tunneling Method and the Measurement Technology[M].Beijing: Science Press, 2002.

[2] Zhang Wenbo,Chen Hongyan. Statistical Analysis of Practical Data and the SPSS12.0 Application [M].Beijing: Posts and Telecom Press,2006.

[3] Liu Zunxiong.Mid-term load forecasting with MPMR [J]. Electric Power,2004,37(9):50-53.

[4] Xu Dongsheng, Yang Wei,Wei Zhe. The Short-time Load Performance Analysis and Forecasting Research Based on SPSS. Power System Protection and Control,2009,37(21):147-151.

[5] Sulem J,Panet M,Guenot A. Closure analysis in deep tunnels[J]. Min Sci \& Geomech Abster,1987,24(3):145-154. 